\title{
Ratio of the expression levels of androgen receptor splice variant 7 to androgen receptor in castration refractory prostate cancer
}

\author{
YOSHITAKA SEKINE, HIROSHI NAKAYAMA, YOSHIYUKI MIYAZAWA, SEIJI ARAI, HIDEKAZU KOIKE, \\ HIROSHI MATSUI, YASUHIRO SHIBATA, KAZUTO ITO and KAZUHIRO SUZUKI
}

Department of Urology, Gunma University Graduate School of Medicine, Maebashi, Gunma 371-8511, Japan

Received September 20, 2020; Accepted September 16, 2021

DOI: 10.3892/ol.2021.13092

\begin{abstract}
In clinical samples, the expression of androgen receptor (AR) and of AR splice variant 7 (AR-V7) is higher in castration-resistant prostate cancer (CRPC) compared with that in hormone-sensitive prostate cancer (PCa). However, there are only a few reports on the ratio of the expression levels of AR-V7 to AR (AR-V7/AR) in prostate tissue. The present study evaluated AR-V7/AR expression in various types of human prostate tissues and CRPC cells. Pretreatment prostate tissue samples from patients with benign prostatic hyperplasia (BPH; $n=18$ ), Gleason score $7(n=17)$, and Gleason score 8-10 $(n=26)$ were collected at the time of prostate biopsy, and tissue samples from CRPC patients $(n=10)$ were collected at the time of transurethral resection of the prostate. Furthermore, androgen-independent LNCaP cells were established. The mRNA expression levels of AR and AR-V7, cell proliferation and prostate-specific antigen (PSA) production were evaluated by reverse transcription quantitative PCR, MTS assay and chemiluminescent enzyme immunoassay, respectively. There was a significant difference in AR-V7/AR expression ratios between the CRPC group and the $\mathrm{BPH}$ and pre-treatment PCa groups (CRPC, 7\%; BPH and pre-treatment $\mathrm{PCa}, 1 \%$ ). Subsequently, we compared the AR and AR-V7 expression levels in CRPC samples with those in the pretreatment prostate tissues from the same patients. The results demonstrated that the AR-V7/AR ratio increased from 3 to $9 \%$ after CRPC onset. Furthermore, in vitro experiment demonstrated that AR-V7 expression in LNCaP cells was
\end{abstract}

Correspondence to: Dr Yoshitaka Sekine, Department of Urology, Gunma University Graduate School of Medicine, 3-9-22 Showa-machi, Maebashi, Gunma 371-8511, Japan

E-mail: ysekine@gunma-u.ac.jp

Abbreviations: PCa, prostate cancer; AR, androgen receptor; CRPC, castration-resistant prostate cancer; $\mathrm{BPH}$, benign prostatic hyperplasia; GS, Gleason score; CS, charcoal-stripped; PSA, prostate-specific antigen; ADT, androgen deprivation therapy; FL, full-length; TURP, transurethral resection of the prostate; CTC, circulating tumor cell; ISUP, International Society of Urological Pathology

Key words: prostate cancer, androgen receptor splice variant 7, castration-resistant prostate cancer, $\mathrm{LNCaP}$ increased after transforming into CRPC cells. The AR-V7/AR ratio also increased from 0.05 to $0.3 \%$. In addition, small interfering (si)-RNA-mediated knockdown of AR inhibited the proliferation of and PSA production from androgen-independent LNCaP cells; however, AR-V7 knockdown had no effect. Conversely, siRNA-mediated knockdown of both AR and AR-V7 inhibited the proliferation of VCAP cells. In summary, the findings from the present study demonstrated that AR-V7 expression and AR-V7/AR ratio were increased after the onset of CRPC, which had a limited role in CRPC cell proliferation. Further investigation is required to clarify the roles of AR other splice variants and AR-V7 in CRPC.

\section{Introduction}

Androgens play an important role in the development of prostate cancer (PCa) (1). Androgen deprivation therapy (ADT) is initially effective in treating most metastatic PCa cases. However, tumors gradually gain androgen-independence, and progress into castration-resistant prostate cancer (CRPC) (2). Androgen receptor (AR) splice variants are involved in one of the mechanisms underlying CRPC $(3,4)$. The AR comprises an N-terminal domain, a DNA-binding domain, a short hinge region and a C-terminal ligand-binding domain (5). AR splice variants, which lack a ligand-binding domain, can activate androgen-independent AR signaling, enabling therefore the survival of tumors against ADT $(3,4)$.

AR-V7 is an AR splice variant $(3,4)$. It contains the $\mathrm{N}$-terminal domain and the DNA-binding domain but lacks other regions. In clinical samples, AR-V7 expression is higher in CRPC cases compared with hormone-sensitive PCa cases and healthy prostate tissues $(4,6)$, and might cause a prostate-specific antigen (PSA) surge after performing radical prostatectomy (3). Furthermore, the detection of AR-V7 in circulating tumor cells (CTCs) in patients with CRPC is associated with enzalutamide and abiraterone resistance (7-9) and increased survival after subjection to taxane therapy than that observed after AR-signal inhibitor treatment (10). VCaP and 22Rv1 PCa cell lines, which are androgen-independent, exhibit higher AR-V7 expression than the LNCaP cell line (4). Furthermore, androgen-independent LNCaP cells cultured under long-term androgen deprivation conditions also express higher levels of AR-V7 than in LNCaP cells (11). These findings suggest the essential role of AR-V7 in CRPC development. 
After hormone sensitive PCa progression into CRPC, the expression of full-length AR (AR-FL or AR) and AR-V7 increases (4). It has been reported that AR-V7 expression is lower than AR-FL in CRPC clinical samples (6); however, studies on the relationship between the AR-V7/AR-FL ratio and CRPC are insufficient. Furthermore, these aforementioned studies have evaluated AR-V7 expression and the AR-V7/AR-FL ratio in different CRPC patients $(4,6)$. It is therefore unclear whether AR-V7 and AR expression levels increase after progression to CRPC or whether the levels are high in patients before cases progress to CRPC.

The present study aimed to determine whether AR-V7 expression would exceed that of AR after transition to CRPC, in clinical samples and PCa cells. AR-V7 and AR expression levels were also compared before and after subjection to ADT in five selected patients. In this study, the efficacy of the therapy targeting AR-V7 in patients with CRPC was investigated.

\section{Materials and methods}

Cell lines and chemicals. The human PCa cell lines, LNCaP and VCAP, were purchased from the American Type Culture Collection. LNCaP cells were cultured in Roswell Park Memorial Institute (RPMI)-1640 Medium (Sigma-Aldrich; Merck KGaA) supplemented with 10\% FBS (Moregate Biotech). LNCaP-low androgen (LA) was defined as an in vitro model of CRPC; these cells were derived from $\mathrm{LNCaP}$ cells cultured in RPMI-1640 medium supplemented with $10 \%$ FBS, charcoal stripped, USDA-approved regions (cat. no. 12676029; Thermo Fisher Scientific, Inc.), which has been absorbed with activated carbon to remove hormones for $>3$ months. VCAP cells were cultured in Dulbecco's modified Eagle's medium (Thermo Fisher Scientific, Inc.), supplemented with 10\% FBS (Moregate Biotech). Rabbit anti-AR polyclonal antibody (cat. no. sc-815), rabbit anti-human $\beta$-actin monoclonal antibody (cat. no. 4967) and mouse anti-AR-V7 monoclonal antibody (cat. no. AG10008) were purchased from Santa Cruz Biotechnology, Inc., Cell Signaling Technology, Inc. and A\&G Pharmaceutical, respectively. When using CS-FBS, cells were cultured in androgen free conditions. All cells were placed at $37^{\circ} \mathrm{C}$ in a humidified incubator containing $5 \% \mathrm{CO}_{2}$.

Prostate sample analysis. Prostate biopsy samples from 61 patients, and transurethral resection of the prostate (TURP) samples from 10 patients were collected at Gunma University Hospital between January 2002 and December 2007, and between January 2012 and December 2013, respectively. All the authors worked as urologists at Gunma University Hospital.

The 61 patients underwent transperineal needle biopsy guided by transrectal ultrasonography. Samples were collected using 18-gauge biopsy cutting needles. Out of all cases, 18 patients presented with benign prostatic hyperplasia (BPH), 17 patients presented with PCa with a Gleason score (GS) (12) of 7 and 26 patients presented with PCa with a GS of 8-10. None of the patients had received therapy before prostate biopsy.

TURP samples were collected from PCa patients after progression to CRPC to improve voiding of urine. All these patients had received ADT before TURP in our hospital. Five patients belonged to both prostate biopsy and TURP groups. The clinical courses of the CRPC patients after TURP were not clear, because some patients were given referrals to local clinics after TURP. Prostate samples were stored in RNAlater ${ }^{\mathrm{TM}}$ Stabilization solution (Qiagen AB) immediately after collection. This study was approved by the Ethical Committee of Gunma University (approval no. 1380).

Reverse transcription quantitative ( $R T-q) P C R$. All prostate samples and cell lysates were analyzed by RT-qPCR. mRNA levels were quantified using the CFX96 Real-Time System (Bio-Rad Laboratories, Inc.). Total RNA was isolated from samples using the Rneasy Mini Kit (Qiagen $\mathrm{AB}$ ) according to the manufacturer's instructions. Complementary DNA (cDNA) synthesis was conducted as previously described (13). TaqMan ${ }^{\mathrm{TM}}$ Reverse Transcription Reagents (Invitrogen; Thermo Fisher Scientific, Inc.; containing MultiScribe ${ }^{\mathrm{TM}}$ Reverse Transcriptase, RNase inhibitor, dNTP mixture, random hexamers and 10X RT buffer) were used for this step. PCR was conducted using $2 \mu \mathrm{l}$ cDNA, $1 \mu \mathrm{l}$ AR primers (cat. no. Hs00171172_m1; Applied Biosystems; Thermo Fisher Scientific, Inc.) or $1 \mu 1$ AR-V7 primers (forward, 5'-CGGAAATGTTATGAACAGGGA TGA-3'; reverse, 5'-CTGGTCATTTTGAGATGCTTGCAAT-3'; probe, 5'-FAM-GGAGAAAAATTCCGGGT-3'), $1 \mu 1 \beta$-actin primers (cat. no. Hs99999903_m1; Applied Biosystems; Thermo Fisher Scientific, Inc.), $10 \mu 1$ of Premix Ex Taq (Takara Bio, Inc.) and $6 \mu \mathrm{l}$ of $\mathrm{mH}_{2} \mathrm{O}$ to prepare a $20-\mu \mathrm{l}$ total reaction system. Subsequently, PCR was performed as follows: One cycle of $10 \mathrm{~min}$ at $95^{\circ} \mathrm{C}$ followed by 40 cycles of $15 \mathrm{sec}$ at $95^{\circ} \mathrm{C}$, and $60 \mathrm{sec}$ at $60^{\circ} \mathrm{C}$. The relative expression levels were normalized to endogenous control $\beta$-actin and were expressed as $2^{-\Delta \Delta C q}(14)$.

Western blotting. Cell lysates were prepared using RIPA buffer (Pierce; Thermo Fisher Scientific, Inc.) supplemented with protease inhibitors (Complete without ethylenediamine tetraacetic acid; Roche Diagnostics $\mathrm{GmbH}$ ) on the ice. Protein concentrations were measured by $\mathrm{DC}^{\mathrm{TM}}$ Protein Assay (Bio-Rad Laboratories, Inc.). Equal amounts of proteins (20-40 $\mu \mathrm{g} / \mathrm{lane})$ were separated by $4-12 \%$ SDS-PAGE and transferred onto nitrocellulose membranes. Membranes were blocked with 5\% skim milk for $1 \mathrm{~h}$ at room temperature and incubated with primary antibodies (rabbit anti-AR polyclonal antibody, 1:500; rabbit anti-human $\beta$-actin monoclonal antibody, 1:1,000; and mouse anti-AR-V7 monoclonal antibody, 1:500) at $4^{\circ} \mathrm{C}$ overnight. Membranes were then incubated with horseradish peroxidase (HRP)-conjugated secondary antibody (1:2,000; anti-rabbit IgG, cat. no. 7074; anti-mouse IgG, cat. no. 7076; Cell Signaling Technology, Inc.) for $1 \mathrm{~h}$ at room temperature. Proteins were visualized using the Immobilon Western HRP Reagent (Millipore, Sigma) by Multi Imager II with MISVS II version 1.0 (BioTools, Inc.) The experiments were repeated three times.

Small interfering (si)-RNA transfection experiments. LNCaP-LA and VCaP cells were seeded into 96-well plates at the density of $6 \times 10^{3}$ cells/well in $100 \mu \mathrm{l}$ culture medium or in 6 -well plates at the density of $3 \times 10^{5}$ cells/well in $1,500 \mu 1$ culture medium and cultured at $37^{\circ} \mathrm{C}$ in a humidified incubator containing $5 \% \mathrm{CO}_{2}$. Then, the cells were transfected with the ON-TARGETplus Non-targeting Pool (cat. no. D-001810-10-05; GE Healthcare Dharmacon, Inc.), ON-TARGETplus AR-exon 7 
siRNA (sense, 5'-UCAAGGAACUCGAUCGAUUUU-3'; antisense, 5'-AUACGAUCGAGUUCCUUGAUU-3'; GE Healthcare Dharmacon, Inc.) or ON-TARGETplus AR-V7 siRNA (sense, 5'-GUAGUUGUGAGUAUSAUGAUU-3'; antisense, 5'-UCA UGAUACUCACAACUACUU-3'; GE Healthcare Dharmacon, Inc.) Each siRNA was transfected at the concentration of $10 \mathrm{nM}$ for LNCAP-LA cells and of $100 \mathrm{nM}$ for VCAP cells using DharmaFECT (GE Healthcare Dharmacon, Inc.). After completion of the transfection for $20 \mathrm{~min}$, cells were incubated for $48 \mathrm{~h}$ at $37^{\circ} \mathrm{C}$ in a humidified incubator containing $5 \% \mathrm{CO}_{2}$.

Cell proliferation assay LNCaP-LA and VCaP cells were seeded into a 96-well plate in $100 \mu \mathrm{l}$ medium supplemented with $10 \% \mathrm{CS}$-FBS and incubated for $24 \mathrm{~h}$ at $37^{\circ} \mathrm{C}$. Cells were subsequently transfected with siRNA as previously mentioned. After incubation at $37^{\circ} \mathrm{C}$ in a $5 \% \mathrm{CO}_{2}$ atmosphere for $144 \mathrm{~h}$, cell proliferation was determined using MTS assay (CellTiter 96 Aqueous one-solution cell proliferation assay; Promega Corporation) according to the manufacturer's instructions.

In vitro measurement of PSA levels. $\mathrm{LNCaP}-\mathrm{LA}$ and $\mathrm{VCaP}$ cells were seeded into 6 -well plates at the density of $3 \times 10^{5}$ cells/well in $1,500 \mu \mathrm{l}$ cultured medium and cultured at $37^{\circ} \mathrm{C}$ in a humidified incubator containing $5 \% \mathrm{CO}_{2}$. Cells were then transfected with siRNA as described previously. After $72 \mathrm{~h}$ incubation, medium was aspirated and cell lysates were prepared using RIPA buffer containing protease inhibitors. PSA concentrations from the cell lysates were evaluated using a One-step sandwich enzyme immunoassay with the E test Tosoh II PSA assay (Tosoh Corp.) according to the manufacturer's instructions. The protein concentration in cell lysates was also determined using a DC protein assay (Bio-Rad Laboratories, Inc.). PSA levels in cell lysates were calculated after dividing the result by the protein concentration as follows; PSA concentration $(\mathrm{ng} / \mathrm{ml}) /$ protein concentration $(\mathrm{mg} / \mathrm{ml})$.

Statistical analyses. All data are expressed as the means \pm standard deviation. Statistical analyses were performed using Wilcoxon signed-rank test for comparisons between two groups or by one-way ANOVA followed by Tukey's post hoc test for comparisons between more than three groups. $\mathrm{P}<0.05$ was considered to indicate a statistically significant difference.

\section{Results}

$A R$ and $A R-V 7$ gene expression in human prostate tissue AR and AR-V7 mRNA levels were determined by RT-qPCR in 61 prostate biopsies and 10 TURP samples. The results demonstrated that AR and AR-V7 expression levels were significantly higher in CRPC samples compared with hormone-sensitive $\mathrm{PCa}$ and $\mathrm{BPH}$ tissues. These results were similar to those reported in a previous study (4) (Fig. 1A). The expression level of AR-V7 was particularly increased by 50 times in CRPC samples compared with the other groups. In addition, the AR-V7/AR ratio was evaluated and was demonstrated to be higher in CRPC samples compared with the other groups (Fig. 1B).

$A R$-V7 gene expression comparison in human prostate tissues of CRPC patients before and after subjection to ADT. There
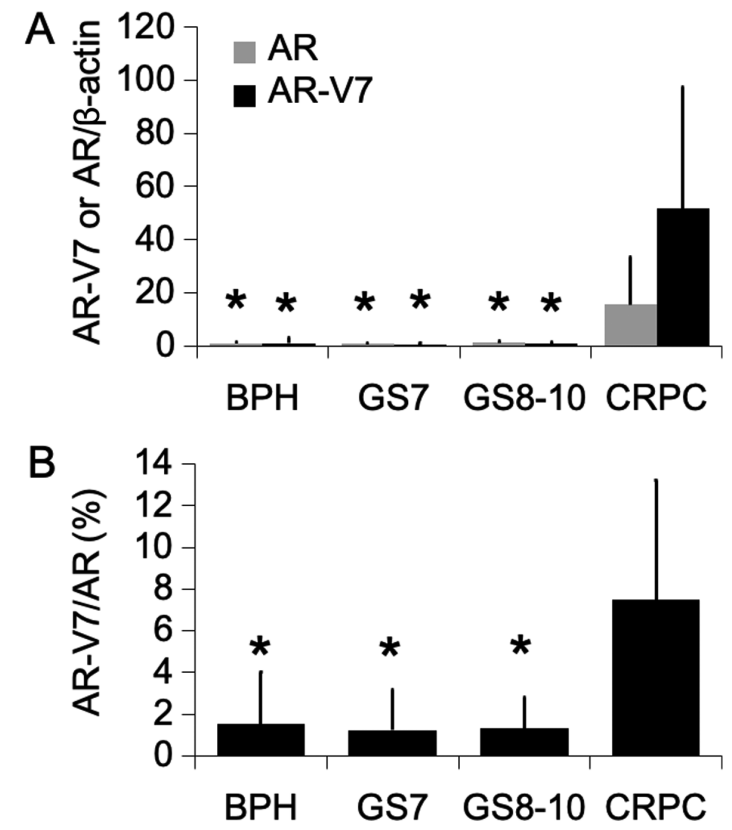

Figure 1. Expression levels of AR and AR-V7 genes in human prostate tissues. (A) mRNA levels of AR and AR-V7 and (B) ratio of AR-V7 to AR in BPH $(n=18)$, GS $7(n=17)$, GS $810(n=26)$ and CRPC $(n=10)$ tissues evaluated by reverse transcription quantitative PCR. Expression levels were expressed as the fold change relative to BPH. Values were expressed as the means \pm standard deviation. ${ }^{*} \mathrm{P}<0.01$ vs. CRPC. AR, androgen receptor; AR-V7, androgen receptor splice variant 7; BHP, benign prostatic hyperplasia; GS, Gleason score; CRCP, castration-resistant prostate cancer.

are few reports comparing the expression of AR-V7 before and after ADT in the same patients. In the present study, we evaluated the change in AR-V7 mRNA expression over time during ADT in samples from the same patient after progression to CRPC (prostate biopsy; before ADT; TURP samples, after ADT). RT-qPCR was performed to evaluate AR and AR-V7 expression levels in prostate tissues obtained from five PCa patients. The results demonstrated that AR-V7 mRNA level was increased by more than 10 times after progression into CRPC in all patients. The expression level of AR also increased in all patients (Fig. 2A). In four patients, the increase in AR-V7 expression level was greater than that for AR. In addition, the AR-V7/AR ratio increased after progression to CRPC in the four patients (Fig. 2B), and the average of AR-V7/AR ratio increased from 3 to $9 \%$ after CRPC onset ( $\mathrm{P}=0.80$; Fig. 2C).

Changes in AR and AR-V7 expression in LNCaP cells after subjection to $A D T$. The effect of androgen deprivation was evaluated on AR-V7 expression in androgen-dependent LNCaP cells. The protein expression levels of AR-V7 could be detected after culturing cells in a medium containing 10\% CS-FBS for a duration of more than 3 months (Fig. 3A). Furthermore, AR-V7 mRNA expression was also increased significantly after culturing the cells in a medium containing $10 \%$ CS-FBS for a duration spanning of more than 3 months (Fig. 3B; P<0.01 vs. LNCaP). However, AR-V7 expression did not increase following culture in the medium containing $10 \%$ CS-FBS for only $48 \mathrm{~h}$ (Fig. 3A and B). The mRNA levels of AR exhibited the same statistical trend as AR-V7 following the same treatments (Fig. 3B; P $<0.05$ vs LNCaP); however, the 

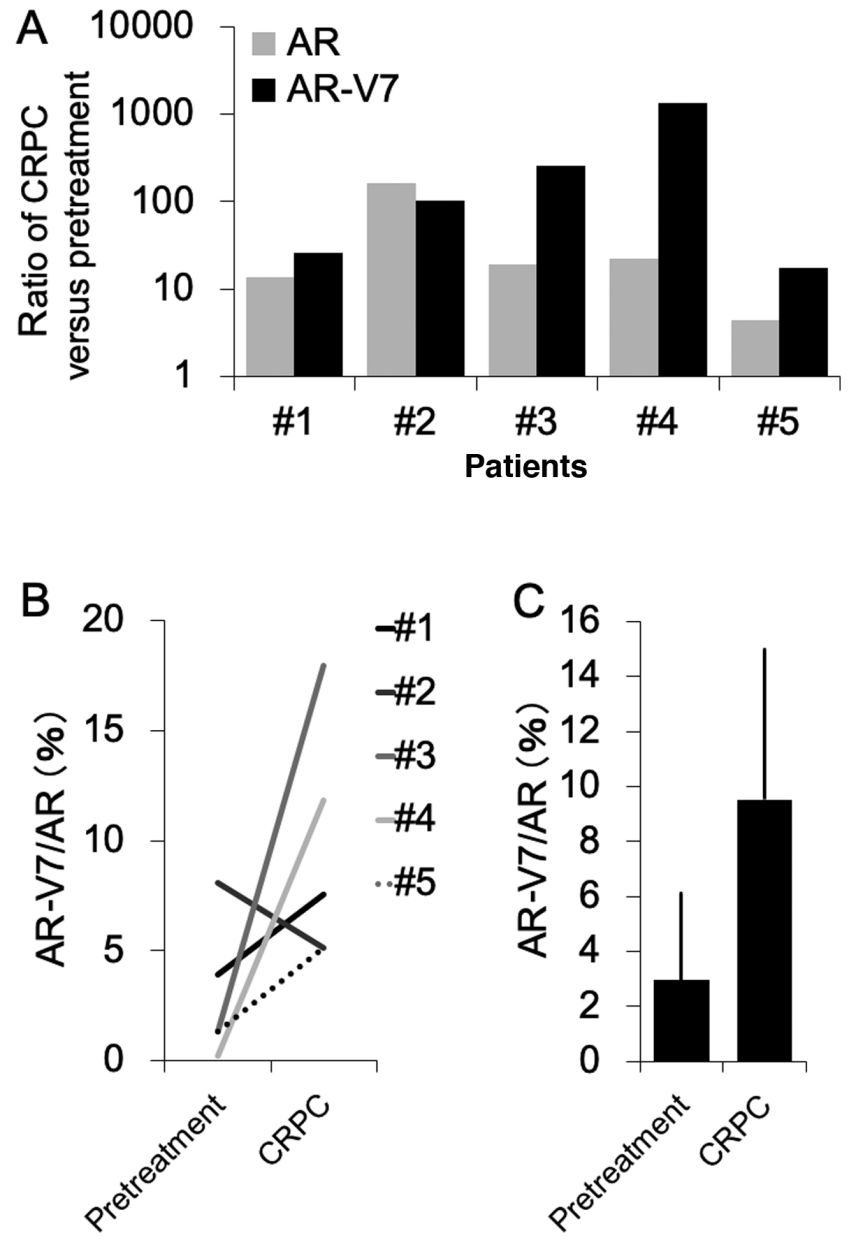

Figure 2. Comparison of AR and AR-V7 gene expression levels in human prostate tissues of CRPC patients before and after subjection to ADT. (A) mRNA expression of AR and AR-V7 and (B and C) ratio of AR-V7 to AR in prostate tissues of five CRPC patients before and after subjection to ADT evaluated by reverse transcription quantitative PCR. Expression values were expressed as the fold change of CRPC relative to pretreatment. Values were expressed as the means \pm standard deviation. ADT, androgen deprivation therapy; AR, androgen receptor; AR-V7, androgen receptor splice variant 7; CRCP, castration-resistant prostate cancer.

protein level did not change within the three groups (Fig. 3A). Subsequently, the AR-V7/AR ratio significantly increased after culturing cells in a culture medium containing $10 \%$ CS-FBS for more than 3 months (Fig. 3C).

Effect of AR-V7 knockdown on cell proliferation and PSA production in LNCAP-LA and VCAP cells. To further determine whether AR-V7 could affect cell proliferation, AR-V7 and AR expression was silenced using siRNA transfections. In the LNCaP-LA and VCAP cells, the expression of AR-V7 and $A R$ proteins was markedly reduced by their respective siRNAs (Fig. 4A). The decrease in AR expression, but not in AR-V7 expression, in LNCaP-LA cells was associated with significantly decreased basal cell proliferation (Fig. 4B) and PSA production (Fig. 4C). Conversely, the knockdown of AR and of AR-V7 inhibited the proliferation of VCAP cells. AR knockdown demonstrated a significantly stronger cell proliferation inhibition compared with AR-V7 (Fig. 4B). There was no decrease in PSA synthesis after AR or AR-V7 knockdown in VCAP cells (data not shown).

\section{Discussion}

The present study demonstrated that AR-V7 expression and the ratio of AR-V7 to AR were higher in CRPC tissues compared with those from hormone-sensitive $\mathrm{PCa}$ and $\mathrm{BPH}$ tissues. Furthermore, AR and AR-V7 expression was found to be higher after progression to CRPC compared with the pretreatment group in the same patient. To the best of our knowledge, the present study is the first highlighting the changes in AR-V7 mRNA expression levels over time during ADT. In addition, inhibition of AR-V7 expression exhibited no effect on LNCaP-LA cell proliferation or PSA production. Taken together, these findings suggested that the expression of AR-V7 and the ratio of AR-V7 to AR may increase after progression to CRPC, and that the effect of AR-V7 expression on CRPC cell viability depends on the cell types.

Novel strategies for CRPC treatment via AR signaling have emerged, and abiraterone and enzalutamide were reported to extend the progression-free survival in CRPC patients $(15,16)$. Furthermore, new inhibitors of AR signaling, including ARN-509 and ODM-201, are under clinical trials for the development of therapeutic approaches for CRPC $(17,18)$. AR signaling plays an important role after $\mathrm{PCa}$ progression to CRPC, and AR splice variants contribute to tumor survival after subjection to ADT $(3,4)$. AR-V7 is one of these variants that lacks a ligand-binding domain. AR signaling is always active without androgens when AR-V7 is present. Clinically, enzalutamide and abiraterone are not effective for CRPC patients with CTCs harboring AR-V7 (7-9). Therefore, detection of AR-V7 in CRPC patients may be considered as a useful strategy for selecting treatments for CRPC patients.

The expression of AR-V7 is usually elevated in CRPC samples $(3,4,19)$. AR-V7 expression is 20 -fold higher in CRPC samples than in hormone-sensitive PCa samples (4). However, only a few reports have compared AR-V7 expression before subjection to ADT and after progression to CRPC in the same patients. Increased AR-V7 expression in human prostate samples obtained from the same patients after progression to CRPC has been reported (19); however, the increase was not evaluated quantitatively. According to RT-qPCR analysis performed in the present study, the mRNA level of AR-V7 was found to be increased $>10$ times after progression to CRPC. A previous study reported that after progression to CRPC, the expression of both AR-V7 and AR are increased (4). In addition, the ratio of AR-V7 mRNA to AR mRNA was demonstrated to be $<1 \%$ after progression to CRPC (6). However, the AR-V7/AR mRNA level ratio is 5-20\% in CTCs from CRPC patients (7). In the present study, the AR-V7/AR mRNA level ratio was $<2 \%$ in $\mathrm{BPH}$ and hormone-sensitive PCa cases and was $\sim 8 \%$ in CRPC. Similar results were obtained at the time of ADT in the same patient's tissue. These data indicated that the increase in AR-V7 mRNA level was greater than that of AR mRNA level after progression to CRPC. The exact clinical function of AR-V7 is not clear; however, it is possible that the increase in AR-V7 expression may serve a biological role in the progression to CRPC.

The expression of AR-V7 increased under the condition of androgen deprivation therapy in androgen-dependent $\mathrm{PCa}$ cells (11). The present study established androgen-independent LNCaP cells and determined the expression of AR-V7 in 
A
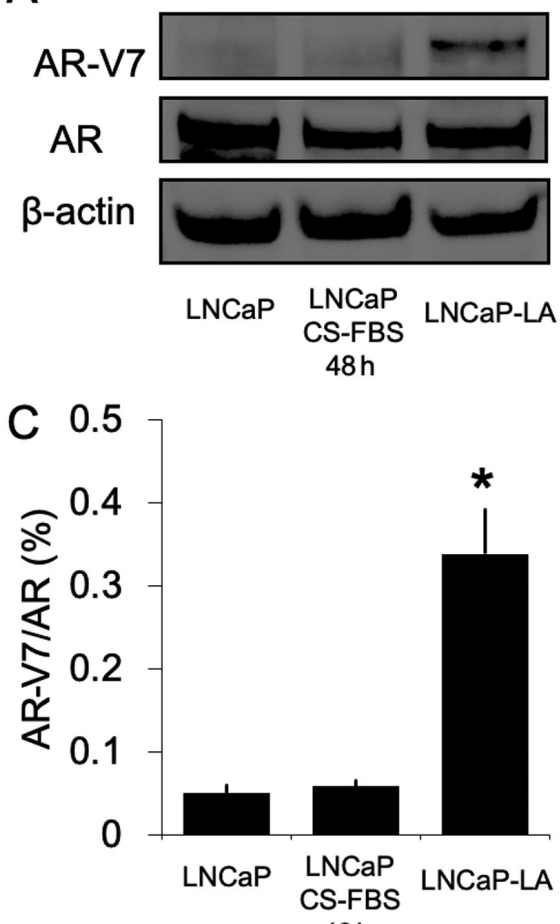

B

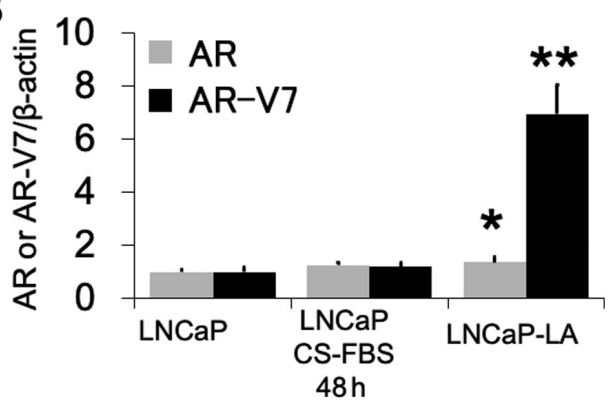

Figure 3. Changes in AR and AR-V7 expression levels after subjection to androgen deprivation therapy in LNCaP cells. LNCaP cells in a medium containing $10 \%$ fetal bovine serum (FBS), LNCaP cells in a medium containing 10\% CS-FBS and LNCaP-LA cells in a medium containing $10 \%$ CS-FBS were incubated for $48 \mathrm{~h}$ before harvesting for (A) western blotting and $(\mathrm{B}$ and $\mathrm{C}$ ) reverse transcription quantitative PCR. Values were expressed as the means \pm standard deviation $(\mathrm{n}=4)$. ${ }^{*} \mathrm{P}<0.05$ vs. LNCaP; ${ }^{* *} \mathrm{P}<0.01$ vs. LNCaP. AR, androgen receptor; AR-V7, androgen receptor splice variant 7; CS-FBS, charcoal-stripped FBS; LA, low androgen.

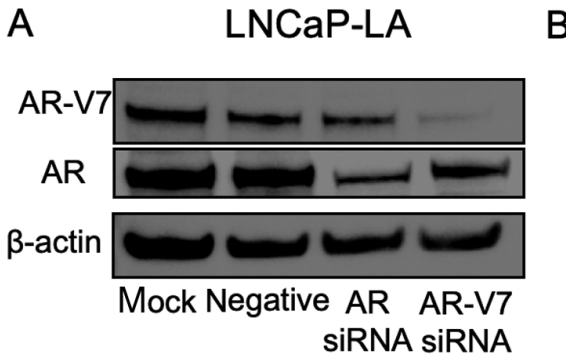
SIRNA SIRNA

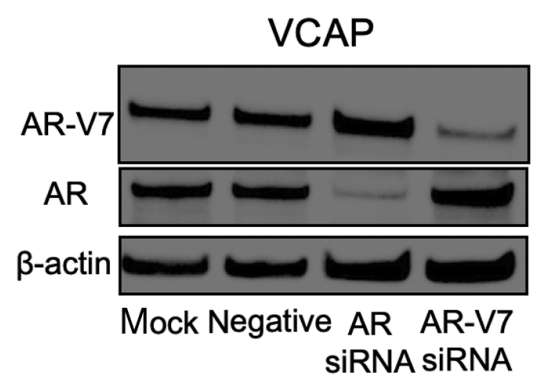

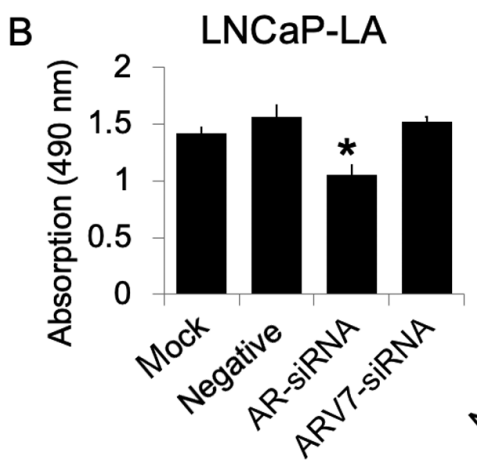

C LNCaP-LA
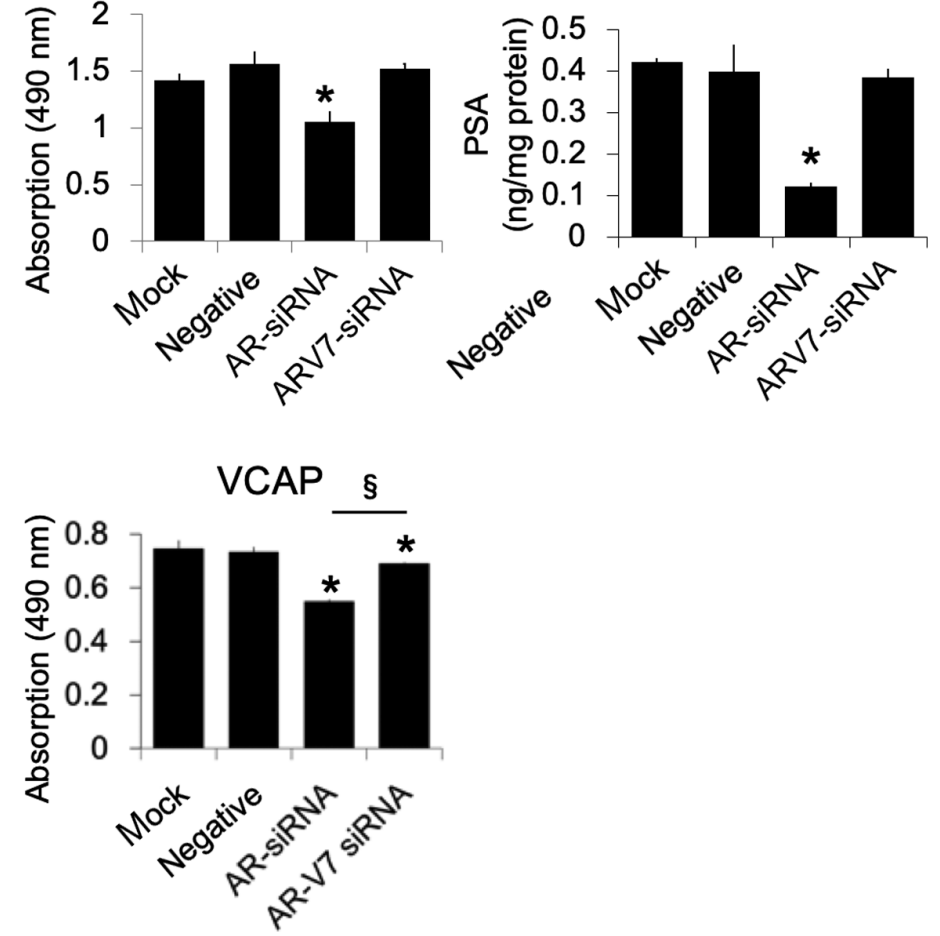

Figure 4. Effect of AR-V7 knockdown on cell proliferation and PSA production in LNCaP-LA and VCAP cells. (A) Mock transfected cells or cells transfected with AR siRNA, AR-V7 siRNA or negative control siRNA were incubated for $48 \mathrm{~h}$ before harvesting for western blotting. (B) After transfection, cells were cultured in a medium supplemented with 10\% CS-FBS. After $144 \mathrm{~h}$, the number of viable cells was evaluated using an MTS assay. Values were expressed as the means \pm standard deviation $(\mathrm{n}=5)$. ${ }^{*} \mathrm{P}<0.05$ vs. negative control siRNA; ${ }^{\circledR} \mathrm{P}<0.01$. (C) After transfection, cells were incubated in a medium containing $10 \%$ CS-FBS. After incubation for $72 \mathrm{~h}$, whole cell lysates were collected for PSA content evaluation. Values were expressed as the means \pm standard deviation $(\mathrm{n}=3)$. " $\mathrm{P}<0.05$ vs. negative control siRNA. AR, androgen receptor; AR-V7, androgen receptor splice variant 7; si, small interfering; PSA, prostate-specific antigen; CS-FBS, charcoal-stripped FBS; LA, low androgen. 
LNCaP-LA cells; however, it was not determined in LNCaP cells. The present study demonstrated that AR-V7 knockdown in LNCaP-LA cells had no effect on cell proliferation or PSA production in the androgen-depleted culture medium. However, a previous study and our present data demonstrated that AR-V7 expression was important for cell proliferation in $\mathrm{VCaP}$ and 22Rvlcells, in which AR-V7 expression was higher than that in androgen-independent LNCaP cells (11). In LNCaP-LA cells, the AR-V7/AR mRNA level ratio was considerably lower than that in human tissue samples. These results indicated that AR-V7 may serve a crucial role in the survival of PCa cells subjected to androgen deprivation; however, the effects of AR-V7 on CRPC progression were dependent on AR-V7 expression levels.

This study presented certain limitations. It has been reported that nuclear-specific AR-V7 protein localization is important for making decisions on CRPC treatment (20). A previous study reported that next-generation androgen deprivation therapies can exert an effect on CRPC via modulating the expression of AR-V7 in CTCs (21). A reason for this is that men with non-nuclear AR-V7 continue to show response to next-generation ADTs (20). The present study evaluated total AR-V7 mRNA level, including both nuclear and non-nuclear AR-V7. We attempted to detect the presence of nuclear AR-V7 proteins in tissue by performing immunohistochemistry using an AR-V7 antibody; however, the results were unsuccessful. In addition, no liquid biopsy was performed in the present study, although a few studies have reported the use of liquid biopsy for detecting the expression of AR-V7 (7,10). Although over 20 AR variants were previously reported (22), the present study only analyzed AR-V7. The roles of other variants should therefore be evaluated in the future. Furthermore, the overall number of analyzed samples was small in the present study, and the use of only five pairs of samples was not sufficient to validate the findings. In addition, we evaluated the relationship between pathological characteristic and the expressions of both AR and AR-V7 in clinical samples without matching the patient cohorts. Regarding pathological evaluation, GS was used in this study, although the International Society of Urological Pathology (ISUP) values were established in 2014 (23). In Japan, ISUP is not common, and it is difficult to reanalyze the samples using ISUP.

In summary, the present study demonstrated that the expression of AR-V7 and the ratio of AR-V7 to AR was increased after progression to CRPC, and that the effects of AR-V7 on CRPC progression depend on AR-V7 expression levels. To the best of our knowledge, this study was the first highlighting the changes in AR-V7 mRNA expression and in the ratios of AR-V7 to AR over time during ADT. Further investigation is required to clarify the roles of AR other splice variants and AR-V7 in CRPC.

\section{Acknowledgements}

The authors would like to thank Ms. Naomi Takase, Ms. Atsuko Oyama and Ms. Hayumi Oyama from the Department of Urology, Gunma University Graduate School of Medicine for their technical assistance.

\section{Funding}

No funding was received.

\section{Availability of data and materials}

The datasets generated during the current study are not publicly available due to ethical restrictions but are available from the corresponding author on reasonable request.

\section{Authors' contributions}

YSe designed the study and drafted the manuscript. YSe, HN and YM performed the cell viability assays and gene expression analysis. SA, HK, HM, YSh and KI contributed to data analysis. YSe and HN confirm the authenticity of all the raw data. KS critically reviewed the manuscript and helped with the experimental design. All authors read and approved the final manuscript.

\section{Ethics approval and consent to participate}

The present study was approved by the Ethical Committee of Gunma University, and written consent was obtained from all enrolled patients for the use of their tissues in analyses. This study was performed in accordance with the principles of the Declaration of Helsinki.

\section{Patient consent for publication}

Not applicable.

\section{Competing interests}

The authors declare that they have no competing interests.

\section{References}

1. Heinlein CA and Chang C: Androgen receptor in prostate cancer. Endocr Rev 25: 276-308, 2004.

2. Scher HI and Sawyers CL: Biology of progressive, castration-resistant prostate cancer: Directed therapies targeting the androgen-receptor signaling axis. J Clin Oncol 23: 8253-8261, 2005.

3. Guo Z, Yang X, Sun F, Jiang R, Linn DE, Chen H, Chen H, Kong X, Melamed J, Tepper CG, et al: A novel androgen receptor splice variant is up-regulated during prostate cancer progression and promotes androgen depletion-resistant growth. Cancer Res 69: 2305-2313, 2009.

4. Hu R, Dunn TA, Wei S, Isharwal S, Veltri RW,Humphreys E, Han M, Partin AW, Vessella RL, Isaacs WB, et al: Ligand-independent androgen receptor variants derived from splicing of cryptic exons signify hormone-refractory prostate cancer. Cancer Res 69: 16-22, 2009.

5. Claessens F, Denayer S, Van Tilborgh N, Kerkhofs S, Helsen C and Haelens A: Diverse roles of androgen receptor (AR) domains in AR-mediated signaling. Nucl Recept Signal 6: e008, 2008.

6. Yu Z, Chen S, Sowalsky AG, Voznesensky OS, Mostaghel EA, Nelson PS, Cai C and Balk SP: Rapid induction of androgen receptor splice variants by androgen deprivation in prostate cancer. Clin Cancer Res 20: 1590-1600, 2014.

7. Antonarakis ES, Lu C, Wang H, Luber B, Nakazawa M, Roeser JC, Chen Y, Mohammad TA, Chen Y, Fedor HL, et al: AR-V7 and resistance to enzalutamide and abiraterone in prostate cancer. $\mathrm{N}$ Engl J Med 371: 1028-1038, 2014.

8. Li Y, Chan SC, Brand LJ, Hwang TH, Silverstein KA and Dehm SM: Androgen receptor splice variants mediate enzalutamide resistance in castration-resistant prostate cancer cell lines. Cancer Res 73: 483-489, 2013.

9. Korpal M, Korn JM, Gao X, Rakiec DP, Ruddy DA, Doshi S, Yuan J, Kovats SG, Kim S, Cooke VG, et al: An F876L mutation in androgen receptor confers genetic and phenotypic resistance to MDV3100 (enzalutamide). Cancer Discov 3: 1030-1043, 2013. 
10. Scher HI, Lu D, Schreiber NA, Louw J, Graf RP, Vargas HA Johnson A, Jendrisak A, Bambury R, Danila D, et al: Association of AR-V7 on circulating tumor cells as a treatment-specific biomarker with outcomes and survival in castration-resistant prostate cancer. JAMA Oncol 2: 1441-1449, 2016.

11. Liu LL, Xie N, Sun S, Plymate S, Mostaghel E and Dong X: Mechanisms of the androgen receptor splicing in prostate cancer cells. Oncogene 33: 3140-3150, 2014.

12. Gleason DF: Classification of prostatic carcinomas. Cancer Chemother Rep 50: 125-128, 1966.

13. Suzuki K, Koike H, Matsui H, Ono Y, Hasumi M, Nakazato H, Okugi H, Sekine Y, Oki K, Ito K, et al: Genistein, a soy isoflavone, induces glutathione peroxidase in the human prostate cancer cell lines LNCaP and PC-3. Int J Cancer 99: 846-852, 2002.

14. Livak KJ and Schmittgen TD: Analysis of relative gene expression data using real-time quantitative PCR and the 2(-Delta Delta C(T)) method. Methods 25: 402-408, 2001.

15. Ryan CJ, Smith MR, Fizazi K, Saad F, Mulders PF, Sternberg CN, Miller K, Logothetis CJ, Shore ND, Small EJ, et al; COU-AA-302 Investigators: Abiraterone acetate plus prednisone versus placebo plus prednisone in chemotherapy-naive men with metastatic castration-resistant prostate cancer (COU-AA-302): Final overall survival analysis of a randomised, double-blind, placebo-controlled phase 3 study. Lancet Oncol 16: 152-160, 2015.

16. Scher HI, Fizazi K, Saad F, Taplin ME, Sternberg CN, Miller K de Wit R, Mulders P, Chi KN, Shore ND, et al; AFFIRM Investigators: Increased survival with enzalutamide in prostate cancer after chemotherapy. N Engl J Med 367: 1187-1197, 2012.

17. Rathkopf DE, Morris MJ, Fox JJ, Danila DC, Slovin SF, Hager JH, Rix PJ, Chow Maneval E, Chen I, Gönen M, et al: Phase I study of ARN-509, a novel antiandrogen, in the treatment of castration-resistant prostate cancer. J Clin Oncol 31: 3525-3530, 2013

18. Fizazi K, Massard C, Bono P, Jones R, Kataja V, James N, Garcia JA, Protheroe A, Tammela TL, Elliott T, et al; ARADES study group: Activity and safety of ODM-201 in patients with progressive metastatic castration-resistant prostate cancer (ARADES): An open-label phase 1 dose-escalation and randomised phase 2 dose expansion trial. Lancet Oncol 15: 975-985, 2014.
19. Yamashita S, Lai KP, Chuang KL, Xu D, Miyamoto H, Tochigi T, Pang ST, Li L, Arai Y, Kung HJ, et al: ASC-J9 suppresses castration-resistant prostate cancer growth through degradation of full-length and splice variant androgen receptors. Neoplasia 14: 74-83, 2012.

20. Scher HI, Graf RP, Schreiber NA, McLaughlin B, Lu D, Louw J, Danila DC, Dugan L, Johnson A, Heller G, et al: Nuclear-specific AR-V7 protein localization is necessary to guide treatment selection in metastatic castration-resistant prostate cancer. Eur Urol 71: 874-882, 2017.

21. Bernemann C, Schnoeller TJ, Luedeke M, Steinestel K, Boegemann M, Schrader AJ and Steinestel J: Expression of AR-V7 in circulating tumour cells does not preclude response to next generation androgen deprivation therapy in patients with castration resistant prostate cancer. Eur Urol 71: 1-3, 2017.

22. Wadosky KM and Koochekpour S: Androgen receptor splice variants and prostate cancer: From bench to bedside. Oncotarget 8: 18550-18576, 2017

23. Epstein JI, Egevad L, Amin MB, Delahunt B, Srigley JR and Humphrey PA; Grading Committee: The 2014 International Society of Urological Pathology (ISUP) Consensus Conference on Gleason Grading of Prostatic Carcinoma: Definition of grading patterns and proposal for a new grading system. Am J Surg Pathol 40: 244-252, 2016.

(c) () $\ominus$ This work is licensed under a Creative Commons cc) ${ }_{\mathrm{EY} \text { NO ND }}$ Attribution-NonCommercial-NoDerivatives 4.0 International (CC BY-NC-ND 4.0) License. 